\title{
Talk Burst Control for Push-to-Talk over Cellular
}

\author{
Meng-Hsun Tsai, Student Member, IEEE, and Yi-Bing Lin, Fellow, IEEE
}

\begin{abstract}
Push-to-talk over Cellular (PoC) is a "walkietalkie"-like service designed for mobile networks. In this service, the speak permission is arbitrated through the talk burst control (TBC) mechanism. This paper proposes an analytic model to study the performance of the TBC mechanism with queueing and without queueing. This analytic model is validated against simulation experiments. Through numerical examples, our study provides guidelines to set up the parameters for PoC service.
\end{abstract}

Index Terms-cellular network, Push-to-talk over Cellular (PoC), talk burst control, Voice over IP (VoIP)

\section{INTRODUCTION}

$\mathbf{P}$ USH-TO-TALK over Cellular (PoC) [1], [2] is a "walkietalkie"-like service designed for mobile networks. In this service, several predefined PoC group members participate in one PoC session. Since the PoC session is half-duplex, only one PoC member speaks at a time, and the others listen. When a PoC member attempts to speak, he/she presses the push-totalk button of his/her mobile terminal to ask for the permission. This mobile terminal installed with the PoC application is called the PoC client. The speak permission is arbitrated through the talk burst control (TBC) mechanism defined in the Open Mobile Alliance (OMA) PoC specifications [3]. Based on the OMA specifications, an Open Service Access (OSA)based PoC system is implemented in National Chiao-Tung University [4], [5].

In the OMA specification, TBC mechanism is implemented by finite state machines (FSMs) in both the client and the server sides. Figs. 1 and 2 illustrate simplified TBC FSMs for PoC server (called $F S M_{G}$ ) and PoC client (called $F S M_{U}$ ), where "U" represents "user" (i.e., for a specific PoC client) and "G" represents "general" (i.e., for the whole group). The prefixes "S:" and "R:" of the transitions represent "send" and "receive", respectively. For a PoC session, there is one $F S M_{G}$ in the PoC server and an $F S M_{U}$ in each of the PoC clients. To clearly describe the PoC procedures, several terms are defined:

- The session initiator is the PoC client who initiates a PoC session.

- An invited PoC client is a PoC group member other than the session initiator.

Manuscript received November 27, 2006; revised February 22, 2007; accepted November 15, 2007. The associate editor coordinating the review of this paper and approving it for publication is H.-H. Chen. The work of Meng-Hsun Tsai was supported by an IBM Ph.D. Fellowship, a Microsoft Fellowship, and a ZyXEL Fellowship. The work of Yi-Bing Lin was supported in part by NSC 96-2752-E-009-005-PAE, NSC 96-2219-E-009-019, NSC 96-2221-E-009-020, Intel, Chung Hwa Telecom, IIS/Academia Sinica ITRI/NCTU Joint Research Center and MoE ATU.

M.-H. Tsai is with the Department of Computer Science, National Chiao Tung University, Taiwan (e-mail: tsaimh@ @csie.nctu.edu.tw).

Y.-B. Lin is with the Department of Computer Science, National Chiao Tung University, Taiwan. He is also with the Institute of Information Science, Academia Sinica, Nankang, Taipei, Taiwan (e-mail: liny@csie.nctu.edu.tw).

Digital Object Identifier 10.1109/LCOMM.2008.060980.

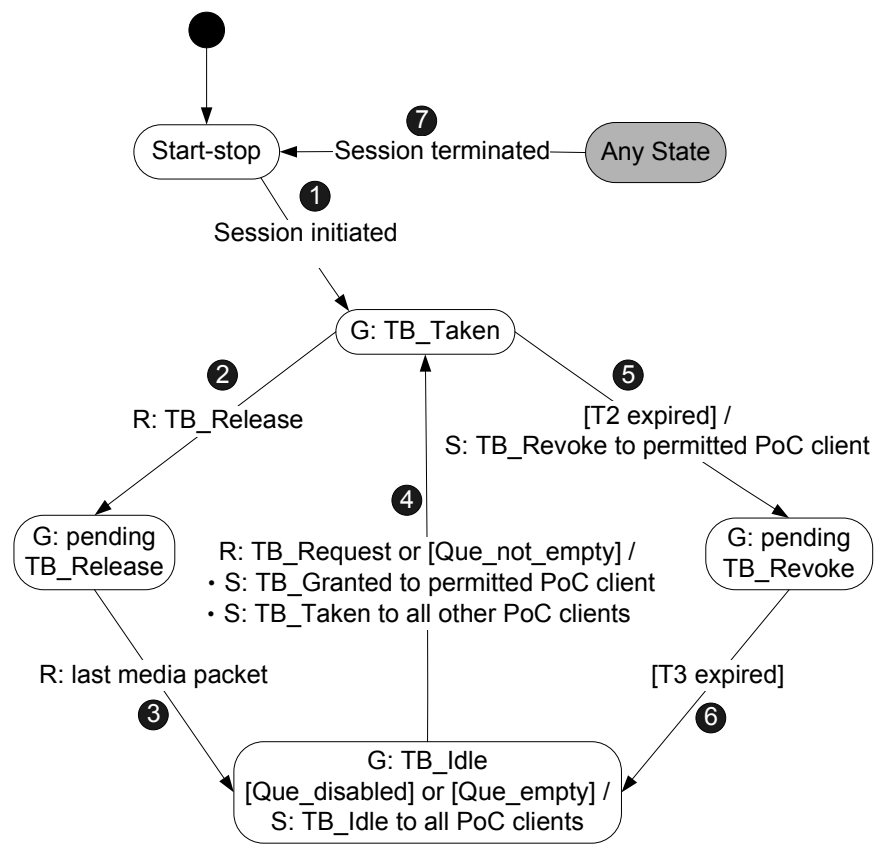

Fig. 1. TBC Finite State Machine for PoC Server $\left(F S M_{G}\right)$.

- A requesting PoC client is a PoC client who requests for speak permission.

- The permitted PoC client is the PoC client who is allowed to speak.

- A listening PoC client is a PoC client who is not permitted to speak.

- A queued PoC client is a PoC client whose request is queued in the PoC server.

A PoC session can be initiated by any group member. Session Initiation Protocol (SIP) [6] and Session Description Protocol (SDP) [7] are utilized for session establishment. To support the PoC service, a new parameter "tb_grant" is added in SDP's attribute field such that PoC server can arbitrate the speak permission during session establishment. If "tb_grant $=1$ ", the $\mathrm{PoC}$ client is granted the permission to speak. Otherwise (i.e., "tb_grant $=0$ "), the $\mathrm{PoC}$ client is not permitted to talk.

PoC is a new service in cellular networks. To our knowledge, all $\mathrm{PoC}$ studies have focused on call setup time and transmission delay of voice packets [8], [9], [10]. These studies did not consider the performance of TBC mechanism. Many TBC-related questions are not answered in these previous studies. These questions include the best setting of revoking time $T_{R}$, the maximum number $N$ of group members that a mobile operator should support, etc. Based on the SIP/Real-time Transport Protocol (RTP) [11] protocols, we 


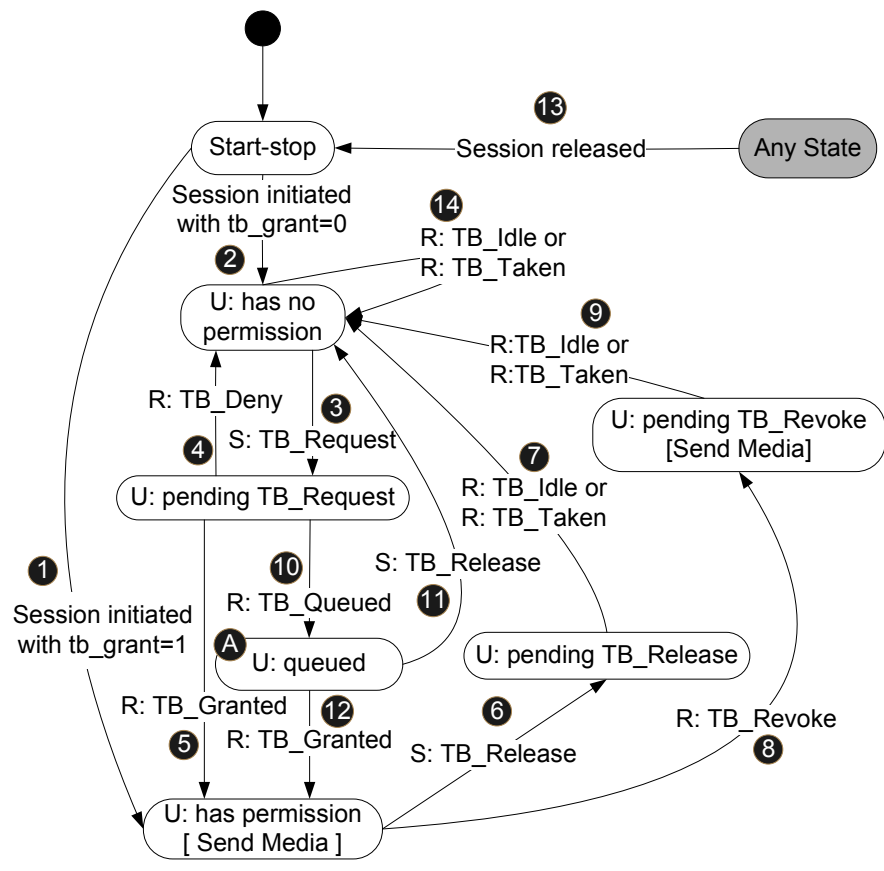

Fig. 2. TBC Finite State Machine for PoC Client $\left(F S M_{U}\right)$.

describe the TBC mechanism, and then propose an analytic model to study the TBC mechanism. Based on our analytic/simulation models, we show how the input parameters affect the performance of the PoC service.

\section{TBC MECHANISM}

For the PoC clients and the PoC server involved in a $\mathrm{PoC}$ session, their FSMs are initialized at the Start-stop state (see Figs. 1 and 2). When the session initiator sends a SIP INVITE message to the PoC server, the PoC server broadcasts SIP INVITE messages with "tb_grant=0" to other group members (the invited PoC clients). Each of the invited PoC clients answers with a SIP $200 \mathrm{OK}$ message and its $F S M_{U}$ enters U: has no permission (transition 2 in Fig. 2). This state means that the PoC client is not permitted to speak.

After receiving the first SIP 200 OK message from the invited PoC clients, the PoC server replies a SIP $200 \mathrm{OK}$ with "tb_grant=1" to the session initiator and $F S M_{G}$ enters G: TB_Taken (transition 1 in Fig. 1). This state means that some PoC client (the session initiator in this case) has obtained the permission. $F S M_{U}$ of the session initiator enters $\mathbf{U}$ : has permission (transition 1 in Fig. 2). This state means that the PoC client is allowed to speak. The session initiator becomes the permitted PoC client, and the invited PoC clients become listening PoC clients. At this moment, the session initiator speaks and all invited PoC clients listen.

After the PoC session is established, each of the PoC clients has built an RTP session with the PoC server. The TBC messages (with the prefix "TB") between the PoC clients and the PoC server are carried by RTP Control Protocol (RTCP) packets [11]. After finishing the talk, the permitted PoC client releases the permission by sending the TB_Release message to the PoC server and its $F S M_{U}$ enters U: pending TB_Release (transition 6 in Fig. 2). In this state, the PoC client stops sending media packets and is waiting for the response from the PoC server. The sequence number of the last delivered media packet is included in the TB_Release message. $F S M_{G}$ enters G: pending TB_Release after receiving the TB_Release message (transition 2 in Fig. 1). In this state, the PoC server keeps forwarding the transient media packets delivered before the TB_Release message is issued from the permitted PoC client. When the last transient media packet has been processed, $F S M_{G}$ enters G: TB_Idle (transition 3 in Fig. 1). This state means that no PoC client is granted the permission to speak. The PoC server broadcasts the TB_Idle message to all PoC clients. $F S M_{U}$ of the permitted PoC client enters $\mathbf{U}$ : has no permission upon receipt of the TB_Idle message (transition 7 in Fig. 2). A listening $\mathrm{PoC}$ client remains in $\mathrm{U}$ : has no permission when it receives the TB_Idle message (transition 14 in Fig. 2). At this point, all PoC clients can compete for the permission to speak.

To obtain the permission, a listening PoC client sends the TB_Request message to the PoC server. The PoC client becomes a requesting $\mathrm{PoC}$ client, where its $F S M_{U}$ enters U: pending TB_Request (transition 3 in Fig. 2). This state means that the PoC client is waiting for the arbitration from the $\mathrm{PoC}$ server. If some other PoC client has been granted the permission, the PoC server sends the TB_Deny message to the requesting PoC client, and $F S M_{U}$ of the requesting PoC client moves back to $\mathbf{U}$ : has no permission (transition 4 in Fig. 2). If the PoC server grants the permission to the requesting $\mathrm{PoC}$ client, it sends the TB_Granted message to this requesting PoC client and the TB_Taken message to other PoC clients. A timer T2 at the PoC server is started; this timer is used to determine whether the permitted PoC client speaks too long (and therefore should be revoked). $F S M_{G}$ enters G: TB_Taken (transition 4 in Fig. 1), and $F S M_{U}$ of the requesting $\mathrm{PoC}$ client enters $\mathbf{U}$ : has permission (transition 5 in Fig. 2). When a listening PoC client receives TB_Taken, its $F S M_{U}$ remains at $\mathbf{U}$ : has no permission, and is not allowed to request for the permission. The requesting PoC client becomes the permitted PoC client.

If the permitted PoC client speaks longer than the T2 timeout period, the PoC server will start timer T3 and send the TB_Revoke message to stop the permitted PoC client. FSM of the permitted PoC client enters U: pending TB_Revoke upon receipt of the TB_Revoke message (transition 8 in Fig. 2). In this state, the PoC client can keep sending media packets until T3 expires. $F S M_{G}$ enters G: pending TB_Revoke (transition 5 in Fig. 1). In this state, the PoC server keeps forwarding media packets until T3 expires. Then $F S M_{G}$ enters G: TB_Idle (transition 6 in Fig. 1). The PoC server sends the TB_Idle message to all PoC clients. $F S M_{U}$ of the permitted $\mathrm{PoC}$ client enters $\mathrm{U}$ : has no permission (transition 9 in Fig. 2) upon receipt of the TB_Idle message. When a listening PoC client receives TB_Idle, its $F S M_{U}$ remains at $\mathbf{U}$ : has no permission. At this point, all PoC clients can compete for the permission to speak.

A queueing option is provided in the TBC mechanism. If this option is selected, then the ungranted requests are buffered in the queue at the PoC server instead of being denied. After the permitted PoC client finishes talking, the PoC server grants the next request from the queue. The state $\mathbf{U}$ : queued (see 
Fig. 2 (A)) in $F S M_{U}$ indicates that a request of the client is queued in the PoC server and will be granted later.

After a PoC client $\mathrm{X}$ has obtained the permission, the PoC server may receive the TB_Request message from another requesting PoC client $\mathrm{Y}$. With the queueing option, $F S M_{G}$ is in the G: TB_Taken state, and $F S M_{U}$ of client $\mathrm{Y}$ is in the U: pending TB_Request state. The PoC server buffers the TB_Request message in the queue and replies the TB_Queued message to client Y. FSM $M_{G}$ stays in G: TB_Taken and $F S M_{U}$ of client $\mathrm{Y}$ enters $\mathbf{U}$ : queued (transition 10 in Fig. 2). The PoC client of a queued request (called queued PoC client) may send the TB_Release message to the PoC server to cancel the request, and its $F S M_{U}$ moves back to $\mathbf{U}$ : has no permission (transition 11 in Fig. 2). In this case, the $\mathrm{PoC}$ server removes the corresponding request from the queue. After the permission is released (or revoked), $F S M_{G}$ will enter G: TB_Idle, and $F S M_{U}$ of the permitted PoC client will enter $\mathbf{U}$ : pending $\mathbf{T B} \_$Release (or $\mathbf{U}$ : pending TB_Revoke). If the queue is not empty, the PoC server processes the next queued request instead of sending out the TB_Idle message. Then the granting procedure is performed at the PoC server. Therefore, the next queued PoC client will receive the TB_Granted message from the PoC server, and its $F S M_{U}$ enters $\mathbf{U}$ : has permission (transition 12 in Fig. 2). This queued PoC client becomes the next permitted PoC client. At the same time, all other PoC clients receive the TB_Taken message from the PoC server. The previously-permitted PoC client becomes a listening $\mathrm{PoC}$ client, and its $F S M_{U}$ enters U: has no permission (transition 7 or 9 in Fig. 2). $F S M_{U}$ of every listening $\mathrm{PoC}$ client remains at $\mathbf{U}$ : has no permission, and $F S M_{U}$ of every queued $\mathrm{PoC}$ client remains at $\mathbf{U}$ : queued.

When a PoC client leaves the PoC session, its $F S M_{U}$ moves back to Start-stop (transition 13 in Fig. 2). The PoC session remains active for other PoC clients. After all $\mathrm{PoC}$ clients leave the PoC session, the PoC session is implicitly terminated. FSM $M_{G}$ moves back to Start-stop (transition 7 in Fig. 1).

\section{AnAlytic Modeling}

This section models the PoC TBC mechanisms with queueing (Approach Q) and without queueing (Approach NQ). Let $N$ be the number of PoC clients in a PoC group. We investigate two timers defined in $F S M_{G}$ : (1) T2 is used to determine whether the PoC client speaks too long; (2) T3 is used to gracefully terminate the talk burst. For simplicity, we define $T_{R}$ as the revoking timer where

$$
T_{R}=T 2+T 3
$$

Three output measures are considered in our study:

- $P_{D}$ : the probability that the request of a PoC client is not granted because the PoC client is not patient in Approach $\mathrm{Q}$ or because the PoC server rejects the request in Approach NQ

- $P_{R}$ : the probability that a PoC client obtains the permission, but is revoked before it finishes the talk

- $W$ (for Approach Q only): the expected waiting time of a request, i.e., the expected time between when a PoC client issues a request and when it is granted the permission to speak or when it leaves the queue without being granted the permission

For a PoC client, we define the following input parameters:

- The inter-request time random variable $\tau_{a}$ with mean $1 / \lambda$

- The speak time random variable $\tau_{s}$ with mean $1 / \mu$ and variance $V_{s}$

- The impatient time random variable $\tau_{i}$ with mean $1 / \omega$ and variance $V_{i}$ (note that a queued PoC client will drop the request if it does not receive the permission within $\left.\tau_{i}\right)$

- The revoking time $T_{R}$ (when $\tau_{s}>T_{R}$, the permitted PoC client is revoked); $T_{R}$ is a fixed period

We assume that $\tau_{a}, \tau_{s}$ and $\tau_{i}$ are exponentially distributed (i.e., $\left.V_{s}=1 / \mu^{2}, V_{i}=1 / \omega^{2}\right)$ in this section. The purpose of analytic model is two folds: to validate the simulation model and to provide mean value analysis. Mean value analysis based on exponential assumptions provides understanding on the "trend" of performance. The validated simulation relaxes the exponential assumptions to accommodate more general (and therefore more practical) scenarios such as those described in [12].

\section{A. Modeling for Approach $Q$}

The PoC TBC mechanism is modeled as a stochastic process. Fig. 3 illustrates the state transition rate diagram of the stochastic process where the state $K=k$ denotes that $k-1$ requests are waiting in the queue at the $\mathrm{PoC}$ server besides the permitted PoC client. In this figure, we define $\gamma(t) h$ to be the probability that the permitted $\mathrm{PoC}$ client is revoked during the interval $(t, t+h)$, where $h \rightarrow 0$. When the process is in state $k$, where $0<k \leq N$, there are $N-k$ listening PoC clients, $k-1$ queued $\mathrm{PoC}$ clients, and a permitted PoC client. State 0 represents that all $\mathrm{PoC}$ clients are listening PoC clients. For any state $k$, there are $N-k$ listening PoC clients that may request for permission to speak. Therefore, the transition rate from state $k$ to state $k+1$ is $(N-k) \lambda$. For any state $k$, where $0<k \leq N$, there are $k-1$ queued PoC clients that may leave the queue with rate $(k-1) \omega$, and the permitted PoC client that may finish the talk with rate $\mu$. Furthermore, the permitted PoC client may be revoked during the interval $(t, t+h)$ with probability $\gamma(t) h$. Therefore, at time $t$ the transition rate from state $k$ to state $k-1$ is $\mu+(k-1) \omega+\gamma(t)$.

Let $\pi_{k}(t)$ denote the probability that the number of requests in the system is $k$ at time $t$. Then based on the state transition rate diagram in Fig. 3, we obtain the following differential difference equations [13]:

$$
\begin{aligned}
\pi_{0}^{\prime}(t)= & \pi_{1}(t)[\mu+\gamma(t)]-\pi_{0}(t) N \lambda \\
\pi_{k}^{\prime}(t)= & \pi_{k-1}(t)(N-k+1) \lambda+\pi_{k+1}(t)[\mu+k \omega+\gamma(t)] \\
& -\pi_{k}(t)[\mu+(k-1) \omega+\gamma(t)+(N-k) \lambda] \\
& \quad(1 \leq k \leq N-1)
\end{aligned}
$$

Under the assumption of statistical equilibrium (i.e., $t \rightarrow \infty$ ), Equations (1) and (2) are re-written as

$$
\begin{aligned}
0= & \pi_{1}(\mu+\gamma)-\pi_{0} N \lambda \\
0= & \pi_{k-1}(N-k+1) \lambda+\pi_{k+1}(\mu+k \omega+\gamma) \\
& -\pi_{k}[\mu+(k-1) \omega+\gamma+(N-k) \lambda] \\
& \quad(1 \leq k \leq N-1)
\end{aligned}
$$




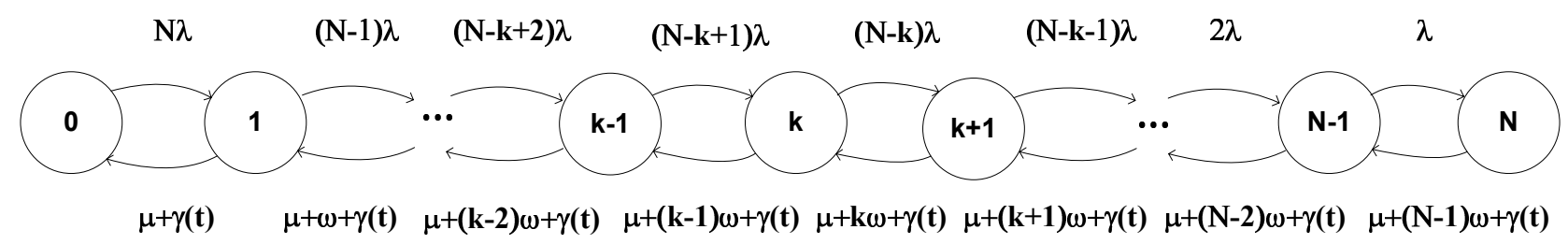

Fig. 3. State Transition Rate Diagram for Approach Q.

where $\pi_{i}=\lim _{t \rightarrow \infty} \pi_{i}(t)$ and $\gamma=\lim _{t \rightarrow \infty} \gamma(t)$ is the revoking rate in equilibrium and will be derived later. Equations (3) and (4) are re-arranged to yield

$$
\begin{aligned}
\pi_{1}= & \frac{\pi_{0} N \lambda}{\mu+\gamma} \\
\pi_{k+1}= & {\left[\frac{\mu+(k-1) \omega+\gamma+(N-k) \lambda}{\mu+k \omega+\gamma}\right] \pi_{k} } \\
& -\left[\frac{(N-k+1) \lambda}{\mu+k \omega+\gamma}\right] \pi_{k-1}
\end{aligned}
$$

From (5) and (6), we have

$$
\pi_{k}=\left\{\frac{\lambda^{k}\left[\prod_{j=0}^{k-1}(N-j)\right]}{\prod_{m=1}^{k}[\mu+(m-1) \omega+\gamma]}\right\} \pi_{0}
$$

where $1 \leq k \leq N$. Since $\sum_{k=0}^{N} \pi_{k}=1$, (7) is solved to yield

$$
\pi_{0}=\left\{1+\sum_{k=1}^{N} \frac{\lambda^{k}\left[\prod_{j=0}^{k-1}(N-j)\right]}{\prod_{m=1}^{k}[\mu+(m-1) \omega+\gamma]}\right\}^{-1}
$$

The revoking rate $\gamma$ is derived as follows. Let $p(\tau, t) h$ be the probability that the elapsed speak time (i.e., the member has talked for the period $\tau$, and the talk has not been finished yet) is between $(\tau-h)$ and $\tau$ (where $h \leq \tau \leq T_{R}$, and $h \rightarrow 0$ ) at time $t$. The probability that the permitted PoC client finishes the talk in any interval $h$ is $\mu h$. Since the elapsed speak time can advance from interval $(\tau-h, \tau)$ to interval $(\tau, \tau+h)$ only if the permitted PoC client does not finish the talk (with probability $1-\mu h)$ during the interval $(t, t+h)$, we have

$$
p(\tau+h, t+h) h=p(\tau, t) h(1-\mu h)
$$

From (9), we have

$$
\frac{p(\tau+h, t+h)-p(\tau, t)}{h}=-\mu p(\tau, t)
$$

Let $h \rightarrow 0$, (10) is expressed as

$$
\frac{\partial p(\tau, t)}{\partial \tau}+\frac{\partial p(\tau, t)}{\partial t}=-\mu p(\tau, t)
$$

When $t \rightarrow \infty, \frac{\partial p(\tau, t)}{\partial t}=0$, and (11) is re-written as

$$
\frac{\partial p(\tau, \infty)}{\partial \tau}=-\mu p(\tau, \infty)
$$

Equation (12) has the general solution

$$
p(\tau, \infty)=A e^{-\mu \tau}
$$

From (13) and since $\int_{0}^{T_{R}} p(\tau, \infty) d \tau=1$, we have

$$
A=\frac{\mu}{1-e^{-\mu T_{R}}}
$$

From (13) and (14), we have

$$
p(\tau, \infty)=\frac{\mu e^{-\mu \tau}}{1-e^{-\mu T_{R}}}
$$

For any interval $(t, t+h)$, a permitted PoC client is revoked if and only if the member has talked for the period $\tau$ at time $t$, where $T_{R}-h<\tau<T_{R}$. Therefore, for any interval $h$ in equilibrium, the revoking probability $\gamma h$ is equal to $p\left(T_{R}, \infty\right) h$. In other words, the revoking rate $\gamma=p\left(T_{R}, \infty\right)$. From (7), (8) and (15), we have

$$
\begin{aligned}
\pi_{k}= & \left\{\frac{\lambda^{k}\left[\prod_{j=0}^{k-1}(N-j)\right]}{\prod_{m=1}^{k}\left[\mu+(m-1) \omega+\frac{\mu e^{-\mu T_{R}}}{1-e^{-\mu T_{R}}}\right]}\right\} \\
& \times\left\{1+\sum_{k=1}^{N} \frac{\lambda^{k}\left[\prod_{j=0}^{k-1}(N-j)\right]}{\prod_{m=1}^{k}\left[\mu+(m-1) \omega+\frac{\mu e^{-\mu T_{R}}}{1-e^{-\mu T_{R}}}\right]}\right\}^{-1}
\end{aligned}
$$

where $1 \leq k \leq N$.

The expected queue length is $E[K]=\sum_{k=0}^{N} k \pi_{k}$. From (16), we have

$$
\begin{aligned}
E[K]= & \left\{\sum_{k=1}^{N} \frac{k \lambda^{k}\left[\prod_{j=0}^{k-1}(N-j)\right]}{\prod_{m=1}^{k}\left[\mu+(m-1) \omega+\frac{\mu e^{-\mu T_{R}}}{1-e^{-\mu T_{R}}}\right]}\right\} \\
& \times\left\{1+\sum_{k=1}^{N} \frac{\lambda^{k}\left[\prod_{j=0}^{k-1}(N-j)\right]}{\prod_{m=1}^{k}\left[\mu+(m-1) \omega+\frac{\mu e^{-\mu T_{R}}}{1-e^{-\mu T_{R}}}\right]}\right\}^{-1}
\end{aligned}
$$

Let $\lambda^{*}$ be the expected request rate to the PoC server. Then

$$
\lambda^{*}=\sum_{k=0}^{N}(N-k) \lambda \pi_{k}=\lambda(N-E[K])
$$

Since a PoC client leaves the system without being granted the permission to speak if its waiting time in the queue is longer than its impatient time, $P_{D}$ can be expressed by

$$
P_{D}=\frac{\begin{array}{c}
\text { the number of requests leaving } \\
\text { the queue due to impatience }
\end{array}}{\text { the number of total request arrivals }}
$$

From (17) and (18), we have

$$
P_{D}=\frac{\sum_{k=1}^{N}(k-1) \omega \pi_{k}}{\lambda^{*}}=\frac{\omega\left\{E[K]-\left(1-\pi_{0}\right)\right\}}{\lambda\{N-E[K]\}}
$$

Similarly, $P_{R}$ can be expressed by

$$
P_{R}=\frac{\text { the number of revoked requests }}{\text { the number of total request arrivals }}
$$


$\mathrm{N} \lambda$

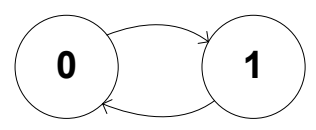

$\mu+\gamma(\mathrm{t})$

Fig. 4. State Transition Rate Diagram for Approach NQ.

From (17) and (19), we have

$$
P_{R}=\frac{\sum_{k=1}^{N} \gamma \pi_{k}}{\lambda^{*}}=\frac{\mu e^{-\mu T_{R}}\left(1-\pi_{0}\right)}{\lambda\{N-E[K]\}\left(1-e^{-\mu T_{R}}\right)}
$$

Let random variable $K_{Q}$ be the number of requests in the queue at the PoC server and random variable $K_{S}$ be the number of permitted PoC clients, then $K=K_{Q}+K_{S}$ and

$$
E[K]=E\left[K_{Q}\right]+E\left[K_{S}\right]
$$

Note that $K_{S}=1$ when system is busy (i.e., some PoC client is speaking), and $K_{S}=0$ when system is idle (i.e., no PoC client is speaking). Therefore,

$$
E\left[K_{S}\right]=1 \times\left(\sum_{k=1}^{N} \pi_{k}\right)+0 \times \pi_{0}=1-\pi_{0}
$$

From Little's result [13], $E\left[K_{Q}\right]$ is expressed as

$$
E\left[K_{Q}\right]=\lambda^{*} W
$$

From (17), (20), (21) and (22), we have

$$
W=\frac{E\left[K_{Q}\right]}{\lambda^{*}}=\frac{E[K]-\left(1-\pi_{0}\right)}{\lambda\{N-E[K]\}}
$$

\section{B. Modeling for Approach $N Q$}

Fig. 4 illustrates the state transition rate diagram for Approach NQ. State $K=0$ represents that no PoC client has the permission, and state $K=1$ represents that a PoC client is speaking. When the stochastic process is in state 0 , there are $N$ listening PoC clients that may request for the permission to speak. Therefore, the transition rate from state 0 to state 1 is $N \lambda$. When the process is in state 1 , the permitted PoC client may finish the talk with rate $\mu$, and may be revoked during the interval $(t, t+h)$ with probability $\gamma(t) h$. Therefore, the transition rate from state 1 to state 0 is $\mu+\gamma(t)$ at time $t$. Similar to the derivation for Approach $\mathrm{Q}$, the following relation at equilibrium is derived:

$$
\pi_{1}=\frac{N \lambda \pi_{0}}{\mu+\frac{\mu e^{-\mu T_{R}}}{1-e^{-\mu T_{R}}}}=\frac{N \lambda\left(1-e^{-\mu T_{R}}\right) \pi_{0}}{\mu}
$$

Since $\pi_{0}+\pi_{1}=1$, (23) is solved to yield

$\pi_{0}=\frac{\mu}{\mu+N \lambda\left(1-e^{-\mu T_{R}}\right)} \quad$ and $\quad \pi_{1}=\frac{N \lambda\left(1-e^{-\mu T_{R}}\right)}{\mu+N \lambda\left(1-e^{-\mu T_{R}}\right)}$

Since a requesting PoC client leaves the system as soon as it finds that the system is busy (i.e., some PoC client is speaking), $P_{D}$ can be expressed by

$$
P_{D}=\frac{\text { number of request arrivals when system is busy }}{\text { number of total request arrivals }}
$$

TABLE I

Comparison of Analytic ANd Simulation Models $\left(V_{s}=1 / \mu^{2}\right.$, $\left.N=10, \omega=\mu, V_{i}=1 / \omega^{2}, T_{R}=3 / \mu\right)$

(a) Approach Q

\begin{tabular}{|c|c|c|}
\hline$\lambda$ & $0.005 \mu$ & $0.05 \mu$ \\
\hline \hline$P_{D}($ Analytic $)$ & 0.0205 & 0.1779 \\
\hline$P_{D}($ Simulation $)$ & 0.0201 & 0.1755 \\
\hline Error & $1.81 \%$ & $1.32 \%$ \\
\hline \hline$P_{R}($ Analytic $)$ & 0.0488 & 0.0409 \\
\hline$P_{R}($ Simulation $)$ & 0.04881 & 0.0410 \\
\hline Error & $0.02 \%$ & $0.24 \%$ \\
\hline \hline$W($ Analytic $)$ & $0.0205 / \mu$ & $0.1779 / \mu$ \\
\hline$W($ Simulation $)$ & $0.0202 / \mu$ & $0.1748 / \mu$ \\
\hline Error & $1.61 \%$ & $1.76 \%$ \\
\hline
\end{tabular}

(b) Approach NQ

\begin{tabular}{|c|c|c|}
\hline$\lambda$ & $0.005 \mu$ & $0.05 \mu$ \\
\hline \hline$P_{D}($ Analytic $)$ & 0.0410 & 0.2995 \\
\hline$P_{D}($ Simulation $)$ & 0.0416 & 0.3003 \\
\hline Error & $1.46 \%$ & $0.27 \%$ \\
\hline \hline$P_{R}($ Analytic $)$ & 0.0477 & 0.0349 \\
\hline$P_{R}($ Simulation $)$ & 0.04775 & 0.03484 \\
\hline Error & $0.10 \%$ & $0.16 \%$ \\
\hline
\end{tabular}

From (17), (24) and (25), we have

$$
P_{D}=\frac{(N-1) \lambda \pi_{1}}{\lambda^{*}}=\frac{(N-1) \lambda\left(1-e^{-\mu T_{R}}\right)}{\mu+(N-1) \lambda\left(1-e^{-\mu T_{R}}\right)}
$$

Similar to the derivation for Approach Q, we derive $P_{R}$ from (17) and (24) as

$$
P_{R}=\frac{\gamma \pi_{1}}{\lambda^{*}}=\frac{\mu e^{-\mu T_{R}}}{\mu+(N-1) \lambda\left(1-e^{-\mu T_{R}}\right)}
$$

The above analytic model is validated against the discrete event simulation experiments. The discrete event simulation model is similar to that described in [14], and the details are omitted. As shown in Table I (where $V_{s}=1 / \mu^{2}, N=10$, $\omega=\mu, V_{i}=1 / \omega^{2}$, and $\left.T_{R}=3 / \mu\right)$, the analytic analysis is consistent with the simulation results.

\section{NUMERICAL EXAMPLES}

Based on the simulation experiments validated against the analytic model, this section investigates the performance of the TBC mechanism. Suppose that $\tau_{a}$ is exponentially distributed with mean $1 / \lambda[15], \tau_{s}$ has the Gamma distribution with mean $1 / \mu$ and variance $V_{s}$, and $\tau_{i}$ has the Gamma distribution with mean $1 / \omega$ and variance $V_{i}$. The Gamma distribution is selected because it has been shown that the distribution of any positive random variable can be approximated by a mixture of Gamma distributions (see Lemma 3.9 in [16]). Following the past experience [17], we can measure the PoC speak times and impatience times from the field and then generate the Gamma distribution from the measured data. In our study, the range for $N$ is selected based on commercial operation. For example, ChungHwa Telecom (CHT) has limited $N$ to 20 (that is, at most 20 members can be defined in a group before the $\mathrm{PoC}$ session is initiated). Also note that finite population with Poisson arrival is widely used in commercial PoC network planning by mobile operators (e.g., FarEasTone or FET [17]) 


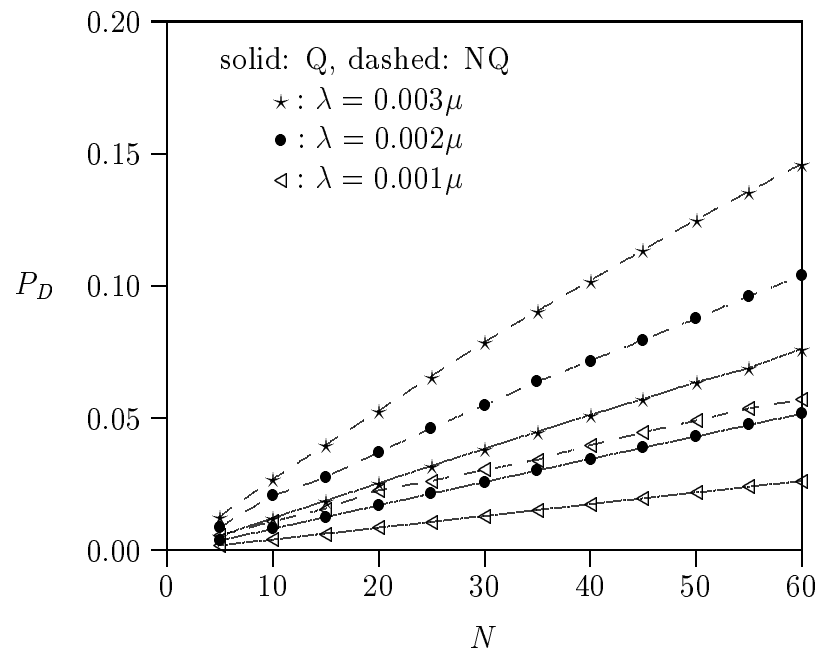

Fig. 5. Effects of $N$ and $\lambda$ on $P_{D}\left(\omega=\mu, V_{i}=1 / \omega^{2}, V_{s}=1 / \mu^{2}\right.$, $\left.T_{R}=3 / \mu\right)$.

and is followed in this paper. The PoC members are allowed to join in and to leave the PoC session at any time during the session, which can be accommodated in the simulation model. The effects of the input parameters are investigated as follows.

Effects of $N$ and $\lambda$ on $P_{D}$ : Under the conditions that $\omega=$ $\mu, V_{i}=1 / \omega^{2}, V_{s}=1 / \mu^{2}, T_{R}=3 / \mu$, Fig. 5 indicates that for the same $P_{D}$ performance, Approach $\mathrm{Q}$ can support twice as many clients as Approach NQ does. For example, when $\lambda=0.003 \mu$, to maintain $P_{D}=0.05$, $N=38$ can be supported in Approach Q and $N=19$ in Approach NQ (therefore, the maximum number $N$ of group members should be set to 38 for Approach $\mathrm{Q}$ and 19 for Approach NQ, respectively). For both Q and NQ approaches, when $\lambda$ increases, the number $N$ supported in the PoC service decreases. For example, to maintain $P_{D}=0.05$ in Approach NQ, $N=51$ can be supported when $\lambda=0.001 \mu, N=27$ when $\lambda=0.002 \mu$, and $N=19$ when $\lambda=0.003 \mu$. We observe that the discrepancy of the $P_{D}$ performance between Approach $\mathrm{Q}$ and Approach NQ decreases as $\omega$ increases. Approach $\mathrm{Q}$ is better than Approach NQ for all $\omega$ values (not shown in this paper). When $\omega=0.5 \mu$, Approach $\mathrm{Q}$ can support 6 times as many clients as Approach NQ does. When $\omega=100 \mu$, Approach Q can only support 1.1 times as many clients as Approach NQ does.

Effects of $\mu$ and $V_{s}$ on $P_{D}$ and $P_{R}$ : In the remainder of this section, we only consider Approach Q. Similar results are also observed in Approach NQ. Fig. 6 indicates that both $P_{D}$ and $P_{R}$ increase and then decrease as $V_{s}$ increases (where $\lambda=0.005 \omega, N=10, V_{i}=1 / \omega^{2}$, and $\left.T_{R}=3 / \mu\right)$. This phenomenon is explained as follows. When the variance $V_{s}$ is small (e.g., $V_{s} \leq 1 / 100 \mu^{2}$ ), all speak times are about the same length. As $V_{s}$ increases, two effects are observed: (I) more speak times longer than $T_{R}$ are observed. These speak times are revoked and result in larger $P_{D}$ and $P_{R}$, (II) more short speak times are also observed, which result in smaller $P_{D}$ and $P_{R}$. As $V_{s}$ increases, Effect (I) is more significant when

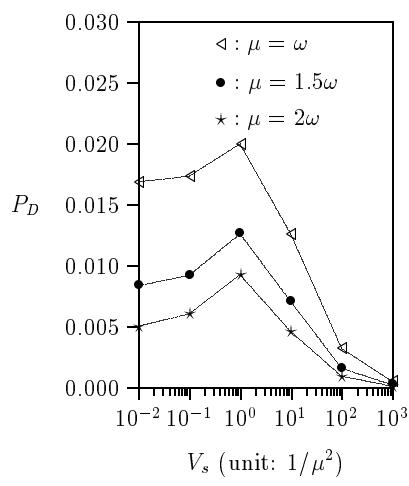

(a) $P_{D}$

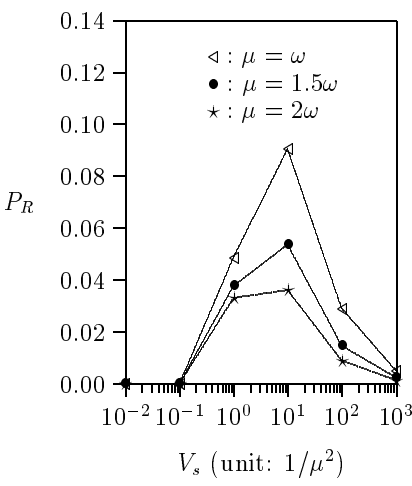

(b) $P_{R}$
Fig. 6. Effects of $\mu$ and $V_{s}$ on $P_{D}$ and $P_{R}$ for Approach Q $(\lambda=0.005 \omega$, $N=10, V_{i}=1 / \omega^{2}, T_{R}=3 / \mu$ ).

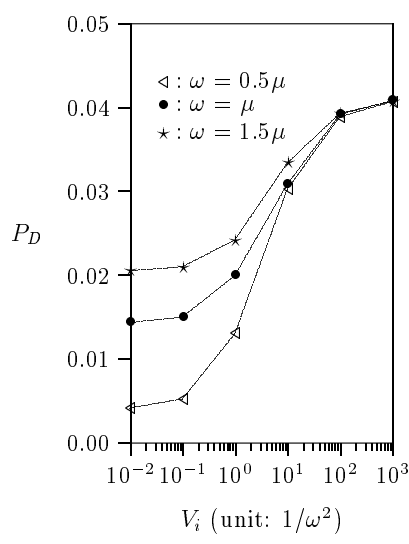

(a) $P_{D}$

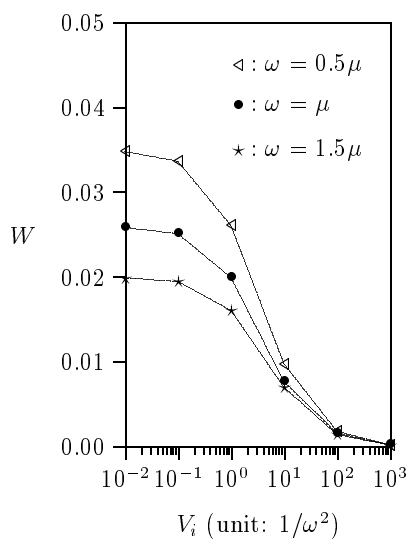

(b) $W$
Fig. 7. Effects of $\omega$ and $V_{i}$ on $P_{D}$ and $W$ for Approach Q $(\lambda=0.005 \mu$, $N=10, V_{s}=1 / \mu^{2}, T_{R}=3 / \mu$ ).

$V_{s}$ is small, and Effect (II) is more significant when $V_{s}$ is large. Therefore, both $P_{D}$ and $P_{R}$ increase and then decrease as $V_{s}$ increases.

Effects of $\omega$ and $V_{i}$ on $P_{D}$ and $W:$ Fig. 7 shows that $P_{D}$ is an increasing function of $V_{i}$, and $W$ is a decreasing function of $V_{i}$ (where $\lambda=0.005 \mu, N=10, V_{s}=1 / \mu^{2}$, and $\left.T_{R}=3 / \mu\right)$. As $V_{i}$ increases, more short and long impatient times are observed. Short impatient times result in larger $P_{D}$ and shorter $W$, while long impatient times result in smaller $P_{D}$ and longer $W$. It is observed that the effect of short impatient times is more significant than long impatient times. Therefore, the net effect is that as $V_{i}$ increases, $P_{D}$ increases and $W$ decreases. The figure also indicates that when $V_{i}$ is very large, $P_{D}$ is not sensitive to the $\omega$ values. That is, the effect of $\omega$ becomes less significant as $V_{i}$ increases.

Effect of $T_{R}$ on $P_{D}$ : Fig. 8 shows that $P_{D}$ increases as $T_{R}$ increases (where $\lambda=0.005 \mu, \omega=\mu, V_{s}=1 / \mu^{2}$, and $\left.V_{i}=1 / \omega^{2}\right)$. For a fixed $P_{D}$, if more clients are supported, a very short revoking time must be enforced to limit the speak times. For example, to maintain $P_{D}=0.021$, the system can support $N=10$ for $T_{R}=4 / \mu$ and $N=30$ for $T_{R}=0.75 / \mu$. 


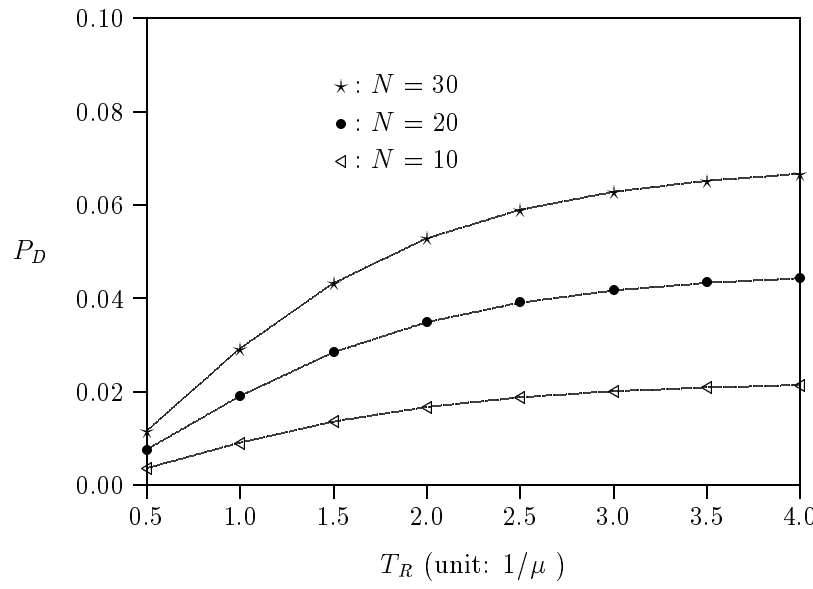

Fig. 8. Effects of $T_{R}$ on $P_{D}$ for Approach Q $(\lambda=0.005 \mu, \omega=\mu$, $\left.V_{s}=1 / \mu^{2}, V_{i}=1 / \omega^{2}\right)$.

\section{CONCLUSIONS}

In PoC service, the speak permission is arbitrated through the TBC mechanism. This paper investigated the performance of the TBC mechanism for PoC service with queueing (Approach Q) and without queueing (Approach NQ). Our study indicates the following:

- As the request rate $\lambda$ increases, the maximum number $N$ of group members can be supported in the PoC service decreases.

- Both the ungranting probability $P_{D}$ and the revoking probability $P_{R}$ increase and then decrease as the variance $V_{s}$ of speak times increases.

- $P_{D}$ is an increasing function of the variance $V_{i}$ of the impatient times. The expected waiting time $W$ of a request is a decreasing function of $V_{i}$. The effect of the impatient rate $\omega$ on $P_{D}$ and $W$ becomes less significant as $V_{i}$ increases.

- For a fixed $P_{D}$, if more clients are supported, a very short revoking time must be enforced to limit the speak times.

\section{REFERENCES}

[1] OMA, Push to talk over cellular (PoC) - architecture, OMA-AD-PoCV2_0_1-20080226-C Candidate Version 2.0 - 26 Feb. 2008.

[2] Y.-B, Lin and A.-C. Pang, Wireless and Mobile All-IP Networks. John Wiley \& Sons, Inc., 2005.

[3] OMA, Push to talk over cellular (PoC) - user plane, OMA-TS-PoCV2_0_1-20080226-C Candidate Version 2.0 - 26 Feb. 2008.
[4] S.-F. Hsu, Y.-C. Lin, Y.-B. Lin, and J.-S. Yang,, "An OSA application server for mobile services," International J. Pervasive Comput. and Commun., vol. 3, no. 1, pp. 102-113, 2007.

[5] L.-Y. Wu, $\mathrm{M}_{\mathfrak{i}}$-H. Tsai, Y.-B. Lin, and J.-S. Yang, "A client-side design and implementation for push to talk over cellular service," Wireless Commun. \& Mobile Comput., vol. 7, no. 5, pp. 539-552, 2007.

[6] IETF, SIP: Session Initiation Protocol, IETF RFC 3261, 2002.

[7] IETF, SDP: Session Description Protocol, IETF RFC 4566, 2006.

[8] A. Brandt, M. Brandt, S. Rugel, and D. Weber, "Admission control for realtime traffic: improving performance of mobile networks by operating on actual throughput," in Proc. IEEE Wireless Communications and Networking Conference (WCNC), New Orleans, LA, Mar. 2005.

[9] P. Kim, A. Balazs, E. van den Brock, G. Kieselinann, and W. Bohm, "IMS-based push-to-talk over GPRS/UMTS," in Proc. IEEE Wireless Communications and Networking Conference (WCNC), New Orleans, Mar. 2005.

[10] E. O'Regan and D. Pesch, "Performance estimation of a SIP based pushto-talk service for $3 \mathrm{G}$ networks," in Proc. European Wireless Conference $(E W)$, Barcelona, Spain, Feb. 2004.

[11] IETF, RTP: A Transport Protocol for Real-Time Applications, IETF RFC 3550, 2003.

[12] Y.-B. Lin, Performance modeling for mobile telephone networks," IEEE Network Mag., vol. 11, no. 6, pp. 63-68, Nov./Dec. 1997.

[13] L. Kleinrock, Queueing Systems: Volume I - Theory. New York: Wiley, 1976.

[14] H.-H. Tsai, A.-C. Pang, Y.-C. Lin, and Y.-B. Lin, "Repacking on demand for speed-sensitive channel assignment," Computer Networks, vol. 47, no. 1, pp. 129-146, 2005.

[15] R. F. Rey, Engineering and Operations in the Bell System. AT\&T Bell Laboratories, 1989.

[16] F. P. Kelly, Reversibility and Stochastic Networks. John Wiley \& Sons, 1979.

[17] FarEasTone Telecom, private communication, 2003.

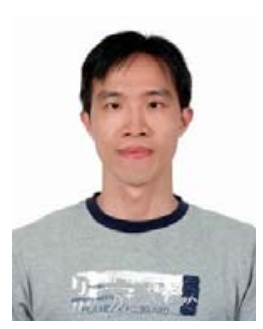

Meng-Hsun Tsai (S'04) received the B.S. and the M.S. degrees from National Chiao Tung University (NCTU), Hsinchu, Taiwan, R.O.C., in 2002 and 2004, respectively. He is currently working toward the Ph.D. degree at NCTU. His current research interests include design and analysis of personal communications services networks, mobile computing and performance modeling.

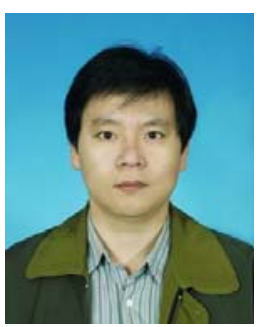

Yi-Bing Lin (M'95-SM'95-F'03) is Chair Professor of Computer Science, National Chiao Tung University. His current research interests include wireless communications and mobile computing. Dr. Lin has published over 220 journal articles and more than 200 conference papers. Lin is the author of the book Wireless and Mobile Network Architecture (coauthor with Imrich Chlamtac; published by John Wiley \& Sons) and the book Wireless and Mobile All-IP Networks (co-author with Ai-Chun Pang; published by John Wiley \& Sons). Lin is an IEEE Fellow, an ACM Fellow, an AAAS Fellow, and an IET(IEE) Fellow. 